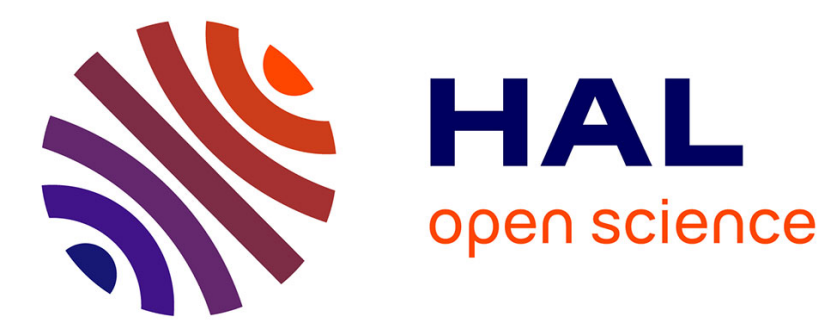

\title{
Un imageur acoustique passif, la "Grande Oreille"
} P. Alais, P. Challande

\section{To cite this version:}

P. Alais, P. Challande. Un imageur acoustique passif, la "Grande Oreille". Journal de Physique IV Proceedings, 1994, 04 (C5), pp.C5-1189-C5-1192. 10.1051/jp4:19945262 . jpa-00252952

\section{HAL Id: jpa-00252952 https://hal.science/jpa-00252952}

Submitted on 1 Jan 1994

HAL is a multi-disciplinary open access archive for the deposit and dissemination of scientific research documents, whether they are published or not. The documents may come from teaching and research institutions in France or abroad, or from public or private research centers.
L'archive ouverte pluridisciplinaire HAL, est destinée au dépôt et à la diffusion de documents scientifiques de niveau recherche, publiés ou non, émanant des établissements d'enseignement et de recherche français ou étrangers, des laboratoires publics ou privés. 


\title{
Un imageur acoustique passif, la "Grande Oreille"
}

\author{
P. ALAIS et P. CHALLANDE
}

Laboratoire de Mécanique Physique, Université Pierre et Marie Curie Paris 6, URA 879 du CNRS, 2 Place de la Gare de Ceinture, 78210 Saint Cyr l'Ecole, France

\begin{abstract}
The "Big Ear" is a signal processing system which permits multiple beamforming of audio signals in real time. It may be used with any kind of arrays of microphones or hydrophones and permits to "ear" a selected point of space through instructions given to the system by a microcomputer. 2000 such points can be defined in the initial phase and shift from one to another may be done in real time so that an acoustical exploration of space is achieved in a short time. An autocalibration of the system obtained from a reference source is also an interesting opportunity. Results obtained with a first realisation which may involve 32 channel arrays, are shown. Aerial and submarine signals have been treated in collaboration with O.N.E.R.A. (Office National d'Etudes et de Recherches Aérospatiales). The potentialities offered by a new system are described. It will be more powerful : 64 sources treated simultaneously from a 64 channel array. The spectral acoustical image will be achieved through an integrated Fast Fourier Transform treatment. The 64 spectral densities of the 64 sources, averaged over a duration of one second will be continuously available at the output.
\end{abstract}

\section{INTRODUCTION}

Le processeur que nous appelons la "Grande Oreille" est un système permettant de traiter en temps réel les signaux acoustiques audibles délivrés par $\mathrm{N}$ microphones ou hydrophones constituant une antenne réseau linéaire ou matricielle, régulière ou aléatoire de géométrie quelconque. Le traitement revient à écouter simultanément $\mathrm{P}$ points (ou directions) de l'espace à partir de voies préformées associées à ces points. Une des originalités du système est la disponibilité instantanée d'un très grand nombre de telles voies préformées et la possibilité de varier, toujours en temps réel, le choix des $\mathbf{P}$ voies retenues, ce qui permet de traquer des points mobiles. La gestion du système est assurée par un micro-ordinateur qui assure le calcul et le chargement des coefficients de retard et d'apodisation du "stock" de voies préformées, lequel peut ainsi être aussi varié indéfiniment en fonction de l'antenne et de l'utilisation.

\section{LA VOIE PREFORMEE NUMERIQUE}

Une voie préformée (figure 1) est classiquement un traitement de recalage temporel des signaux $R_{n}$ délivrés par une source située en $M_{p}$ aux microphones $M_{n}$, lesquels sont retardés du temps de vol $\left(M_{p} M_{n} / c=r_{p n} / c\right)$ par rapport à la source. Ces retards peuvent être importants, mais diffèrent peu entre eux, et le traitement adapté consiste en général à apporter au signal le retard complémentaire: $\tau_{p n}=\left(r_{p o}-r_{p n}\right) / c$ qui assure à tous les signaux le retard commun $r_{p o} / c$ et les recale entre eux. Il est important par ailleurs d'affecter aux divers signaux des coefficients de pondération $\beta_{\mathrm{n}}$ constituant 
"l'apodisation" de la voie préformée dont le rôle est d'apodiser la zone focale ainsi réalisée autour de $\mathbf{M}_{\mathrm{p}}$ en éliminant, autant qu'il est possible, les lobes secondaires. On peut finalement écrire le signal reçu par le microphone $M_{n}$ de la source $M_{p}$ :

$$
R_{n}(t)=\alpha_{n} S_{p}\left(t-r_{p n} / c\right)
$$

et le signal d'écoute reconstruit de la zone $M_{p}$ :

$$
R_{p}(t)=\sum_{n} \beta_{n} \alpha_{n} R_{n}\left(t-\tau_{p n}\right)=\left(\sum_{n} \beta_{n} \alpha_{n}\right) S_{p}\left(t-\tau_{p o}\right) \quad \tau_{p o}=r_{p o} / c
$$

Naturellement, on privilégie ce faisant la source $S_{p}$, tout en réduisant la contribution des sources qui se trouvent ailleurs. L'efficacité de la réduction constitue la qualité de la focalisation acoustique ainsi réalisée, ce dont nous ne discuterons pas ici.

La version numérique de la voie préformée consiste à utiliser les échantillons $R_{n i}$ des signaux $R_{\mathbf{n}}$ numérisés aux temps $t_{\mathbf{i}}$ avec une récurrence d'échantillonnage $\Delta t$. Ces signaux sont stockés dans une mémoire tournante de I échantillons. Quand l'échantillon $R_{n i}$ est écrit dans la mémoire au temps $t_{i}$, on dispose des $I$ valeurs contenues dans la mémoire : $R_{n i}$, où $i '$ varie de $\mathrm{i}-\mathrm{I}+1$ à $\mathrm{i}$. La formation d'un signal représentatif de la voie préformée $M_{p}$ peut être constituée par l'addition correctement pondérée des échantillons lus dans les $\mathrm{N}$ mémoires associées aux signaux $\mathbf{R}_{\mathbf{n}}$ et choisis de telle sorte que le retard numérique $[i \sim(i-I+1) \Delta t]$ (décompté à partir de l'écriture la plus antérieure possible) approche au mieux le retard $\tau_{p n}$.

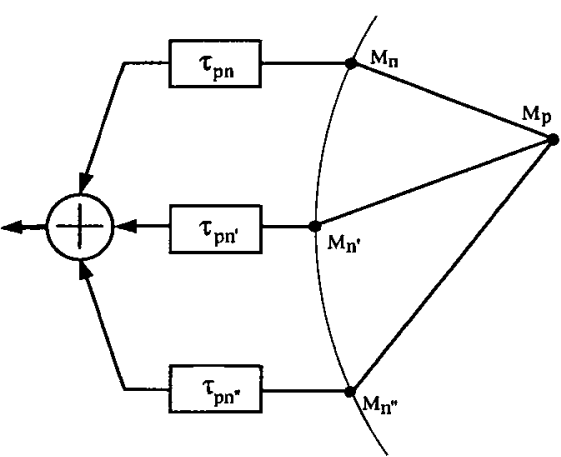

Figure 1

L'erreur maximale commise sur ces retards est fixée par la récurrence d'échantillon $\Delta t$ qui vaut $8 \mu$ s dans les systèmes réalisés. Cette valeur correspond à une erreur de distance d'environ $3 \mathrm{~mm}$ dans l'air et de 12 $\mathrm{mm}$ dans l'eau, et n'a pas d'influence sensible sur les résultats dans les applications pratiques.

\section{LA PREMiere Grande Oreille}

Rien d'original dans ce qui précède. Les voies préformées numériques sont utilisées classiquement en acoustique sous-marine et de plus en plus en échographie médicale ou industrielle. L'intérêt des systèmes décrits ici réside dans la gestion effectuée en temps réel par un microordinateur du choix de $\mathrm{P}$ voies préformées que l'on peut calculer en apparence simultanément du fait de la rapidité des circuits numériques. Ceci permet "limagerie acoustique" en temps réel d'une source distribuée éventuellement mobile. La première "Grande Oreille" traite $\mathrm{N}=32$ canaux de réception. Elle peut délivrer en temps réel un échantillon de voie préformée en $8 \mu \mathrm{s}$, soit une voie préformée au dit échantillonnage, ou par exemple 4 voies préformées à l'échantillonnage de $32 \mu \mathrm{s}$, avec une sortie multiplexée ou bien toute combinaison du même type. Les 32 voies de réception sont numérisées à 12 bits par 4 convertisseurs analogiquesnumériques affectés chacun à 8 canaux multiplexés analogiquement à la fréquence de $1 \mathrm{MHz}$. Les échantillons numériques sont stockés dans 32 sections d'une seule mémoire à 32 × 2048 mots de 12 bits. Ceci permet la relecture d'échantillons avec une panoplie de retards allant jusqu'à 2048 x $8 \mu$ s (de l'ordre de $16 \mathrm{~ms}$ ), soit environ $5 \mathrm{~m}$ dans l'air ou $24 \mathrm{~m}$ dans l'eau, et laisse beaucoup de latitude pour la géométrie des antennes. Toutes les opérations de formation de voies sont effectuées par un seul multiplieuraccumulateur travaillant sur 16 bits à la cadence de $8 \mathrm{MHz}$. Les valeurs des retards et des coefficients d'apodisation affectés aux échantillons $R_{n i}$ proviennent de mémoires préalablement écrites à partir d'un micro-ordinateur. Lors de la phase d'initialisation, celui-ci fournit et charge les informations relatives à 2048 voies préformées. 


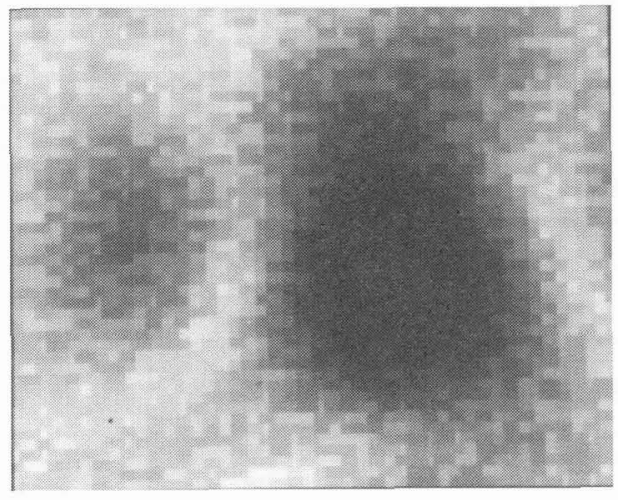

(a)

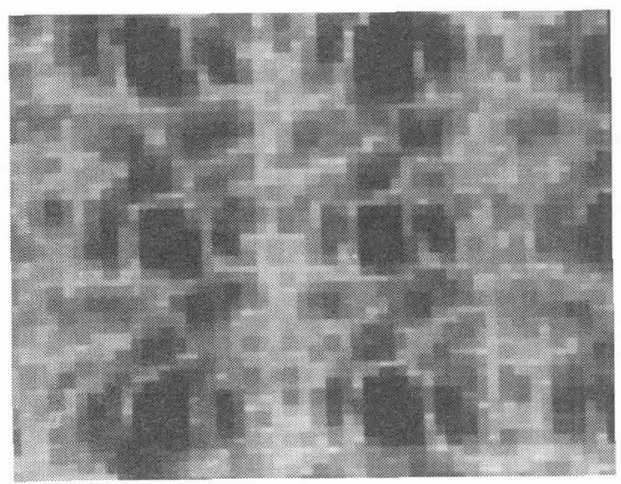

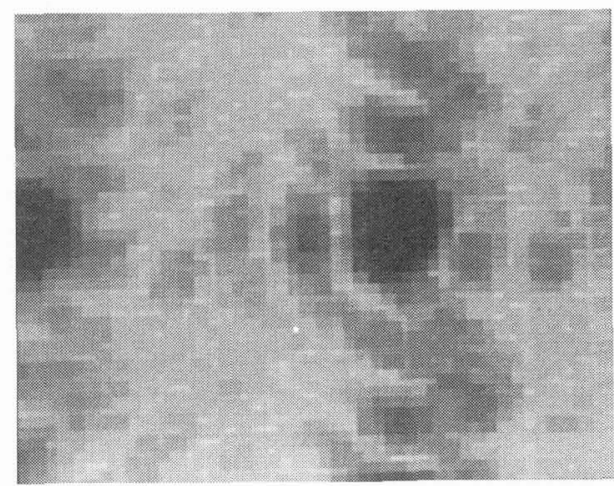

(b)

\section{Figure 2}

Image apparente d'une source ponctuelle harmonique située à $1 \mathrm{~m}$ vue par une rétine de 6 par 5 microphones (pas : $10 \mathrm{~cm}$ ) focalisant dans le plan source, pour différentes fréquences: $2 \mathrm{kHz}$ (a), $6 \mathrm{kHz}$ (b) et $10 \mathrm{kHz}$ (c).

(c)

Il est à noter que l'on peut remplacer la phase d'étalonnage, opération souvent fastidieuse avec les systèmes classiques par une procédure automatique : 32 "voies préformées" sont affectées à la réception pure et simple de chaque canal pris isolément, le système enregistre la réception obtenue à partir d'une source de référence ponctuelle et, en tenant compte des résultats, corrige automatiquement les niveaux reçus par des coefficients d'apodisation adaptés. A la suite de quoi, le système peut imager une source distribuée fixe ou mobile en visant séquentiellement ou de façon semi-parallèle un certain nombre de ses points. La figure 2 montre par ailleurs l'évolution classique de l'image apparente d'une source ponctuelle harmonique en fonction de la fréquence "vue" à partir d'une antenne matricielle de $5 \times 6$ microphones au pas de $10 \mathrm{~cm}$. Par ailleurs, l'ONERA [1,2] a pu montrer l'intérêt de ce système en post-traitant des signaux du passage d'un sous-marin enregistrés par un polygone d'écoute. La figure 3 est l'image fournie par une maquette du T.G.V. disposée dans la soufflerie CEPRA 19 (étude O.N.E.R.A. à l'instigation de la S.N.C.F.). 


\section{Figure 3}

Image de bruit obtenue sur une maquette simplifiée du T.G.V. dans la soufflerie CEPRA 19. On note que l'intensité est maximale au niveau du pantographe.

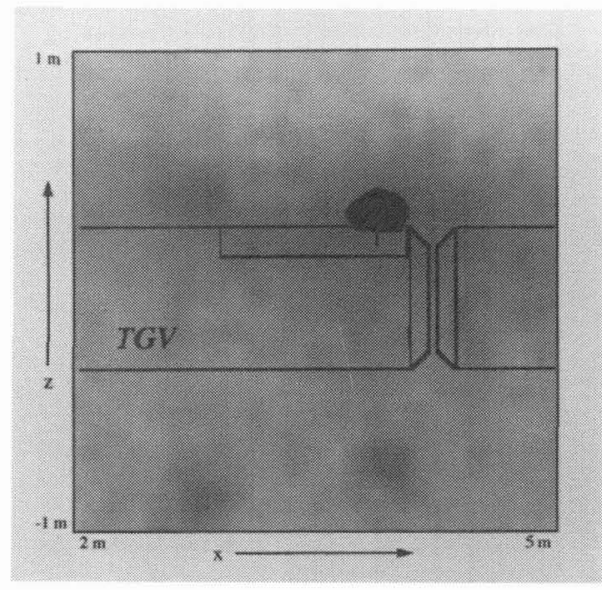

\section{La NOUVElle Grande Oreille}

Ce nouveau système, tout en gardant les avantages du précédent, autorise l'utilisation de 64 canaux de réception. Il forme, grâce à un traitement parallèle, chaque échantillon de voie préformée en $1 \mu$ s et est donc 16 fois plus puissant que le précédent. Parmi tous les fonctionnements possibles, le mode imageur consiste à utiliser une tranche de 64 voies préformées obtenues en parallèle avec un échantillonnage de $64 \mu \mathrm{s}$. Cette valeur correspond à la limite de Shannon de $7,8 \mathrm{kHz}$, ce qui est adapté à beaucoup d'applications concernant le bruit. La reconstruction en temps réel de cette tranche permet d'associer les 64 points retenus à des points attachés à une structure mobile et donc d'imager une telle source.

Une autre originalité importante de ce système est de disposer d'un système de mémoire tampon permettant de traiter l'énorme flot de données résultant $\left(10^{6}\right.$ mots de 16 bits par seconde) par une transformation de Fourier ultra rapide réalisée en électronique dédiée. On calcule ainsi pour chaque voie préformée retenue de la densité spectrale moyennée sur 64 passes de 256 échantillons successifs, c'est-àdire sur un peu plus d'une seconde. Avec ce type de fonctionnement, l'appareil délivre chaque seconde 64 courbes de densités spectrales moyennées sur 128 raies de 0 à $7,8 \mathrm{kHz}$. Ce flot de données est parfaitement acceptable pour le micro-ordinateur et ses moyens de stockage numérique. Par ailleurs, l'écoute permanente d'une voie préformée est possible et obtenue à partir d'un signal interpolé numériquement selon Shannon avec un échantillonnage de $4 \mu \mathrm{s}$.

\section{Conclusion}

Les systèmes décrits plus haut présentent l'intérêt de permettre limagerie passive en temps réel ou quasi réel de sources acoustiques distribuées avec la possibilité de suivre un telle source dans son mouvement. La première "Grande Oreille" est plutôt adaptée à l'exploration de l'espace visé par balayage séquentiel. Le deuxième système offre naturellement l'écoute en parallèle sur 64 points que l'on peut également déplacer en temps réel. Enfin, le traitement de Fourier intégré dans le système permet d'obtenir une image spectrale des mêmes 64 points moyennée sur une seconde.

\section{REFERENCES}

[1] G. Fournier, P. Alais \& G. Elias : "Noise measurements with phased or delayed focused arrays," International Noise and Vibration Control Conference, Saint-Petersburg, May 31-June 3, 1993.

[2] G. Foumier, G. Elias, J.F. Piet, F. Putcrabey, P. Bertrand \& A. Jacques : "Localisation d'images de bruit d'écoulement sur maquettes en soufflerie," Actes du 13ème colloque d'aéro- et hydro-acoustique, (SFA-AAAF) Lyon, 9-11 juin 1993. 